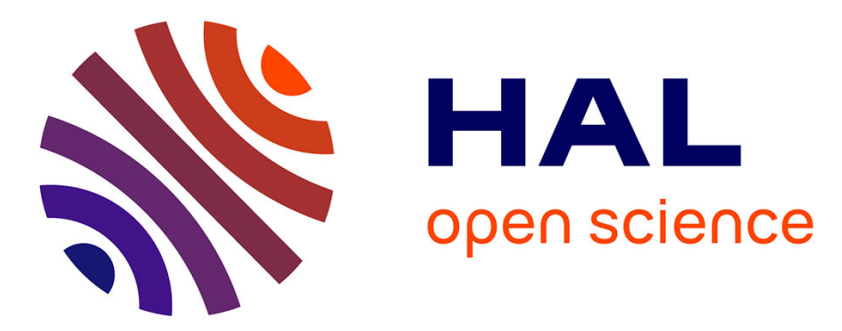

\title{
A level set based approach for the finite element simulation of a forming process involving multiphysics coupling: Ultrasonic welding of thermoplastic composites
}

\author{
A. Levy, S. Le Corre, N. Chevaugeon, A. Poitou
}

\section{To cite this version:}

A. Levy, S. Le Corre, N. Chevaugeon, A. Poitou. A level set based approach for the finite element simulation of a forming process involving multiphysics coupling: Ultrasonic welding of thermoplastic composites. European Journal of Mechanics - A/Solids, 2011, 30 (4), pp.501. 10.1016/j.euromechsol.2011.03.010 . hal-00753960

\author{
HAL Id: hal-00753960 \\ https://hal.science/hal-00753960
}

Submitted on 20 Nov 2012

HAL is a multi-disciplinary open access archive for the deposit and dissemination of scientific research documents, whether they are published or not. The documents may come from teaching and research institutions in France or abroad, or from public or private research centers.
L'archive ouverte pluridisciplinaire HAL, est destinée au dépôt et à la diffusion de documents scientifiques de niveau recherche, publiés ou non, émanant des établissements d'enseignement et de recherche français ou étrangers, des laboratoires publics ou privés. 


\section{Accepted Manuscript}

Title: A level set based approach for the finite element simulation of a forming process involving multiphysics coupling: Ultrasonic welding of thermoplastic composites

Authors: A. Levy, S. Le Corre, N. Chevaugeon, A. Poitou

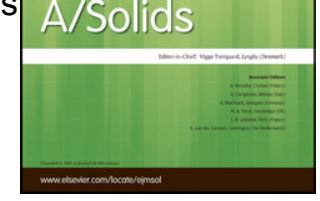

PII: $\quad$ S0997-7538(11)00044-1

DOI: $\quad$ 10.1016/j.euromechsol.2011.03.010

Reference: EJMSOL 2696

To appear in: European Journal of Mechanics / A Solids

Received Date: 11 June 2010

Revised Date: 21 March 2011

Accepted Date: 23 March 2011

Please cite this article as: Levy, A., Le Corre, S., Chevaugeon, N., Poitou, A. A level set based approach for the finite element simulation of a forming process involving multiphysics coupling: Ultrasonic welding of thermoplastic composites, European Journal of Mechanics / A Solids (2011), doi: 10.1016/ j.euromechsol.2011.03.010

This is a PDF file of an unedited manuscript that has been accepted for publication. As a service to our customers we are providing this early version of the manuscript. The manuscript will undergo copyediting, typesetting, and review of the resulting proof before it is published in its final form. Please note that during the production process errors may be discovered which could affect the content, and all legal disclaimers that apply to the journal pertain. 


\section{A level set based approach for the finite element simulation of a forming process involving multiphysics coupling: ultrasonic welding of thermoplastic composites}

Arthur Lévy : arthur.levy@ec-nantes.fr

Steven Le Corre : steven.le-corre@ec-nantes.fr, +33 240272527 (work) / +33240272566 (fax)

Nicolas Chevaugeon : nicolas.chevaugeon@ec-nantes.fr

Arnaud Poitou : arnaud.poitou@ec-nantes.fr

Address : GeM - Institut de genie civil et de mécanique, 1 rue de la Noë, 44321 Nantes cedex 3, France.

Dear Editor,

We would like to publish this work, entitled "A level set based approach for the finite element simulation of a forming process involving multiphysics coupling: ultrasonic welding

of thermoplastic composites.", in European Journal of Mechanics A/Solids which seems to us one of the best quality review in the concerned field.

The paper proposes an original set of numerical methods to simulate the process of ultrasonic welding of thermoplastic composite plates, based on the time homogenization technique we have proposed in a former paper (still in press). The time scale separation implies the solving of three coupled problems, two "macro-temporal" ones describing the long-time thermal and mechanical evolution during the squeezing of the interfacial polymer, and a "micro-temporal" one describing the self-heating due to vibrations.

This paper details the adopted numerical methods based on the use of level set function in an Eulerian framework and presents some validation tests of the developed FEM code. It also presents some physical analyses of the process that enable a better understanding of the ultrasonic welding.

I really hope this article will receive all your attention and match the scope and the quality requirements of EJM-A.

Yours sincerely,

Steven Le Corre. 


\title{
A level set based approach for the finite element simulation of a forming process involving multiphysics coupling: ultrasonic welding of thermoplastic composites.
}

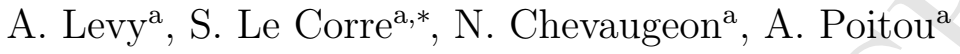 \\ ${ }^{a} G e M$ - Institut de genie civil et de mécanique, 1 rue de la Noë, 44321 Nantes, France.
}

\section{Abstract}

Thermoplastic composite materials offer new perspective in the mechanical industry, especially in aeronautic. The assembly of huge structures by welding is made possible by the ability of the matrix to melt. This paper focuses on ultrasonic welding process where heating is confined at the welding interface. This is achieved thanks to a local mechanical dissipation in triangles specially located at the interface, called energy director. In order to better understand the phenomena that occur at the energy director scale, we propose to model and simulate the polymer flow at the interface. Based on a previous work, the flow under vibration is modeled using three coupled boundary value problems. A specific simulation tool is then developed for solving those three problems. It entails specific numerical methods: a level set method allows to handle the large geometry change, and an iterative

\footnotetext{
*corresponding author

Email addresses: arthur.levy@ec-nantes (A. Levy), steven.le-corre@ec-nantes (S. Le Corre), nicolas.chevaugeon@ec-nantes (N. Chevaugeon), arnaud.poitou@ec-nantes (A. Poitou)
} 
solver manages the multiphysical aspects. The novel simulation obtained is validated with a qualitative comparison to experiments. Then, an analysis of the numerical results allows to understand the phenomena that enables welding. A thermomechanical localization heats the tip of the energy director. This initiates a fold of polymer, that progressively fills the gap between the two plates to weld, and ensures conditions for adhesion.

Keywords: numerical simulation, multiphysical modeling, forming process, level set, ultrasonic welding

\section{Introduction}

For some decades now, composite materials tend to replace traditional metallic structures in the industry. Their specific properties make them very competitive compared to metals, especially in the aeronautic industry. Although thermoset matrix composites are traditionally used in the industry, for several years now, thermoplastic composites open new prospects. Besides their good physical and chemical properties, new forming processes may allow to produce huge parts. In order to assemble those parts, welding processes are made possible by the ability to melt the matrix. In this paper, we focus on an original welding process for thermoplastic composites: the ultrasonic welding.

In this process, in order to achieve a local heating of the interface, triangular bulges, called energy directors, are molded on one of the plates to be welded. The assembly is then positioned under a tool called sonotrode, which applies simultaneously an ultrasonic harmonic loading at twenty kilohertz and a constant compression loading on the directors, which first heat 


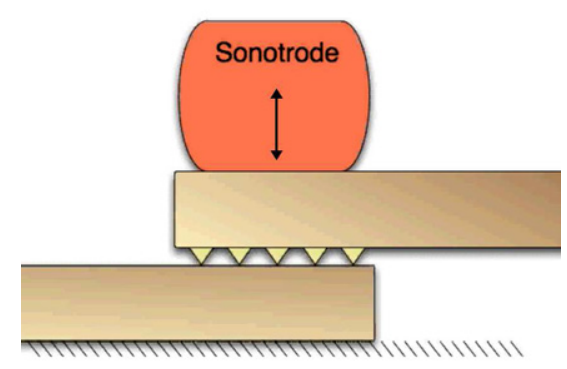

(a) Assembly.
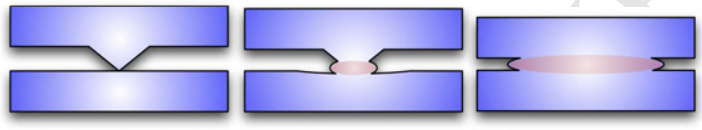

(b) Flow at the interface.

Figure 1: Principle of the ultrasonic welding technique.

because of the viscous dissipation and then flow at the interface until welding is achieved (see Fig. 1).

Different studies of the process focus on the so called mesoscopic scale of the energy director and the welding zone. Tolunay et al. (1983) proposed an experimental study of the heating and bonding mechanism. Benatar and Gutowski (1989) described the process in five successive steps: vibrations, heating, heat transfer, flow and healing. Benatar et al. (1989), followed by Nonhof and Luiten (1996), modeled the viscoelastic heating in the energy director for different polymers. Many authors have underlined the major importance of the geometry of the director on the process. Based on the viscoelastic analysis, Wang et al. (2006) and Suresh et al. (2007) simulated with finite element method the temperature field induced by different energy director shapes and dimensions. Even Benatar et al. (1989), was aware of the importance of the energy director shape and considered a so called multimass lumped parameter model to model the triangular shape. The heating turns out to be initiated thanks to the special geometry of the director, that fulfills a concentrator role. Nevertheless none considered simultaneously the 
vibration and the change of geometry in the welding zone. Yet as soon as the polymer goes past a glassy state, it deforms irreversibly, so that the thermal source calculation is not suited anymore.

In a previous paper (Levy et al., 2010) we fully considered theoretically this double scale loading consisting of simultaneous vibration and squeezing of the director, based on a small strain viscoelastic model. Thanks to a time homogenization method, the vibrating flow was described by three single scale boundary value problems: an elastic (harmonic) problem giving the short time self heating, a thermal problem giving the average heating in time, both being coupled to a macro-temporal flow problem giving the director's shape evolution. Thanks to a simple uniform test case validating the relevance and efficiency of such an approach, it could also be proved that a rectangular director would not heat enough. Accounting for the geometry is now an essential issue.

In the present article, basing on that theoretical work, we propose to simulate the process with a more industrial geometry, namely a triangular energy director. Only a numerical resolution may allow to solve the boundary value problems on this geometry. Accordingly, a specific finite element tool is developed especially designed to handle the two particularities of the retained modeling: the multiphysical aspect and the large geometry changes. The multiphysical aspect is treated using an iterative algorithm. An Eulerian framework with level sets allows to handle the large geometry changes.

In the first section, we propose a modeling of the flow at the interface thanks to three coupled problems: an elastic problem describes the effects of the vibration, fluid mechanics describes the geometry change and a thermal 
problem with a specific source term describes the temperature evolution. In the second section, the implementation of original numerical methods in a new finite element software is presented. It allows to simulate the effect of ultrasonic vibration on the flow of polymer. This simulation is qualitatively validated in section 4 . Then, in the last section, a discussion shows that this simulation allows to better understand the process and highlights some limitations of the numerical methods.

\section{Modeling of the process}

\subsection{Domain}

Considering the "static" welding case, where the sonotrode does not advance, we consider a bidimensional domain $\Omega$ containing one energy director and the two composite plates. Plane strain assumption is retained. Thanks to the symmetries, the domain is reduced to a half director and a half step between two directors, as shown in Fig. 2. Composite plates are described with a first homogeneous material (aimed at describing their transverse behaviour). The energy director and the two superficial layers of the plates are described thanks to a second material called the polymer. Lastly, simulations also require the use of a third material model for the surrounding air.

\subsection{Boundary value problem}

A time homogenization analysis was previously proposed by Levy et al. (2010). This rigorous mathematical analysis allowed the complex thermoviscoelastic behaviour of the polymer at the interface to be modelled using three coupled boundary value problems. This idea is retained in the proposed modeling, as shown in Fig. 3. Nonetheless some liberties are taken concern- 


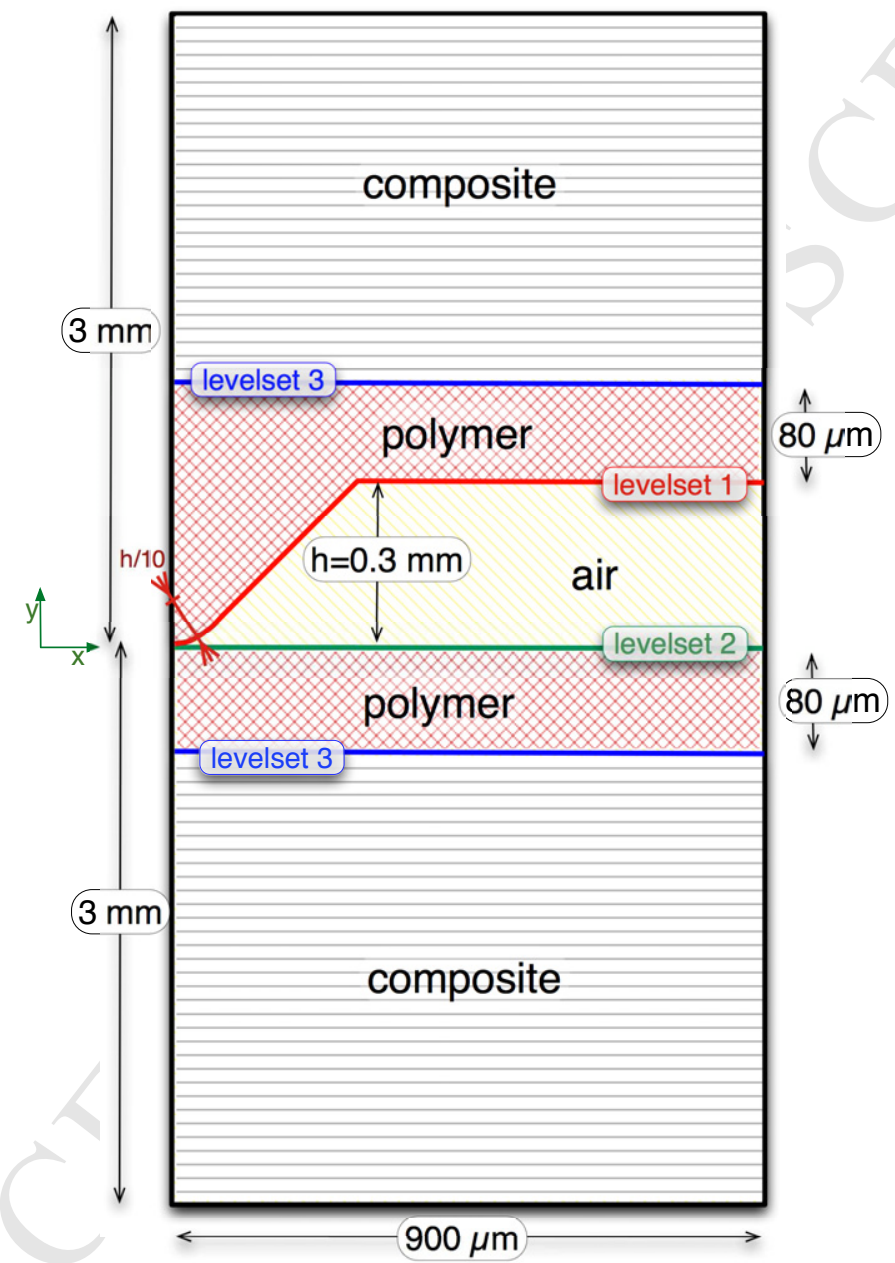

Figure 2: Domain and materials, not to scale. 


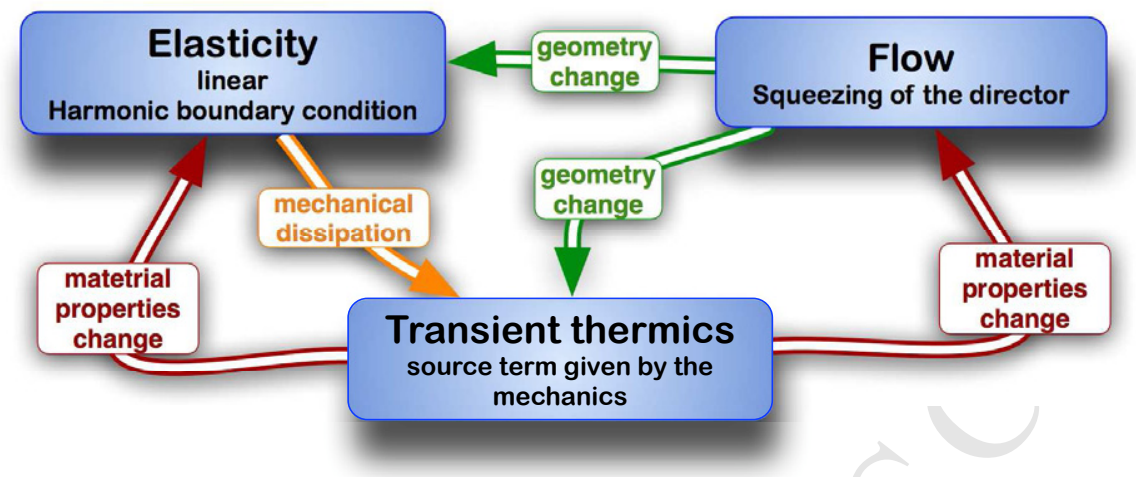

Figure 3: Modeling of the thermomechanical problem using three coupled boundary value problems.

ing the constitutive laws. These differences allow to use more realistic models for each of the three physics, regarding the material behaviours.

\subsubsection{Elasticity}

An elastic problem (e) is described by the usual elasto-static equations, under small strain assumption:

$$
\left\{\begin{array}{l}
\boldsymbol{\sigma}_{e}=\lambda \operatorname{Tr}(\boldsymbol{\varepsilon}) \boldsymbol{I}+2 \mu \boldsymbol{\varepsilon} \text { on }(\Omega) \\
\boldsymbol{\nabla} \cdot \boldsymbol{\sigma}_{e}=\mathbf{0} \text { on }(\Omega)
\end{array},\right.
$$

with the boundary conditions

$$
\left\{\begin{array}{l}
\boldsymbol{u}=\boldsymbol{a} \text { on }\left(\Gamma_{\text {sup }}\right) \\
\boldsymbol{u}=\mathbf{0} \text { on }\left(\Gamma_{0}\right) \\
\boldsymbol{\sigma}_{e} \cdot \boldsymbol{n}=0 \text { on }\left(\Gamma_{\text {lat }}\right)
\end{array} .\right.
$$

In these equations $\boldsymbol{\sigma}_{e}$ denotes the Cauchy stress tensor, $\lambda$ and $\mu$ the two Lamé coefficients, and $\varepsilon$ the strain tensor. It describes the effect of the ultrasonic vibration imposed by the tool at the associated short time. The 
elastic behaviour of the composite under small strain is compressible with a Poisson ratio of 0.4 (see below, table 1). The static assumption corresponds to the near-field welding (Grewell et al., 2003) where the dimensions of the parts to weld are far smaller than the wave length (which is around $10 \mathrm{~cm}$ for the transverse direction of the composite).

A vertical harmonic displacement $\boldsymbol{a} \sin (\omega t)$ describing the vibration of the tool is imposed on the upper boundary. Since the problem is linear, it is solved in amplitude only. The real sonotrode vibration amplitude is around $5 \times 10^{-5} \mathrm{~m}$. Nevertheless, a value of $\|\boldsymbol{a}\|=2 \times 10^{-5} \mathrm{~m}$ was chosen so that the simulation fits the experimental observations. This value is slightly lower than expected. This can be explained by the hammering effect of the sonotrode on the upper plate (Nonhof and Luiten, 1996) that was not taken into account. Vertical displacement is imposed to zero on the lower boundary (see Fig. 4a).

\subsubsection{Fluid mechanics}

The flow problem (f) reads:

$$
\left\{\begin{array}{l}
\boldsymbol{\sigma}_{v}=2 \eta \boldsymbol{D}-p \boldsymbol{I} \text { on }(\Omega) \\
\boldsymbol{\nabla} \cdot \boldsymbol{\sigma}_{v}=\mathbf{0} \text { on }(\Omega) \\
\boldsymbol{\nabla} \cdot \boldsymbol{v}=0 \text { on }(\Omega)
\end{array}\right.
$$

with the boundary conditions

$$
\left\{\begin{array}{l}
\boldsymbol{\sigma}_{v} \cdot \boldsymbol{n}=\overline{\boldsymbol{s}} \text { on }\left(\Gamma_{\text {sup }}\right) \\
\boldsymbol{v}=\mathbf{0} \text { on }\left(\Gamma_{i}\right) \\
\boldsymbol{\sigma}_{v} \cdot \boldsymbol{n}=0 \text { on }\left(\Gamma_{\text {lat }}\right)
\end{array}\right.
$$

where $\boldsymbol{D}=\boldsymbol{\nabla}_{s} \boldsymbol{v}$ is the strain rate tensor, $\boldsymbol{\sigma}_{v}$ the Cauchy stress tensor, $\boldsymbol{\Sigma}_{v}$ the extra-stress, and $p$ the pressure, unknown because of the incompressibility 


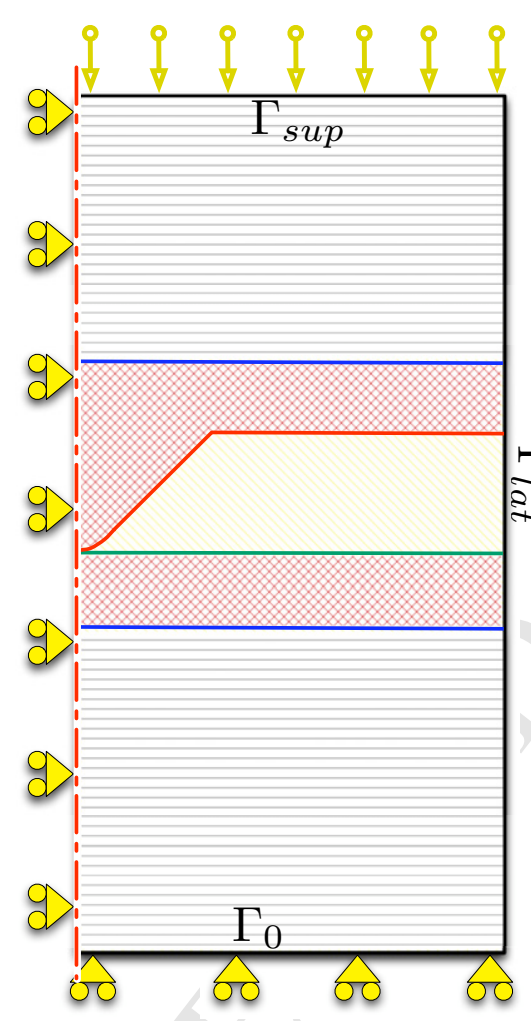

(a) Elasticity.

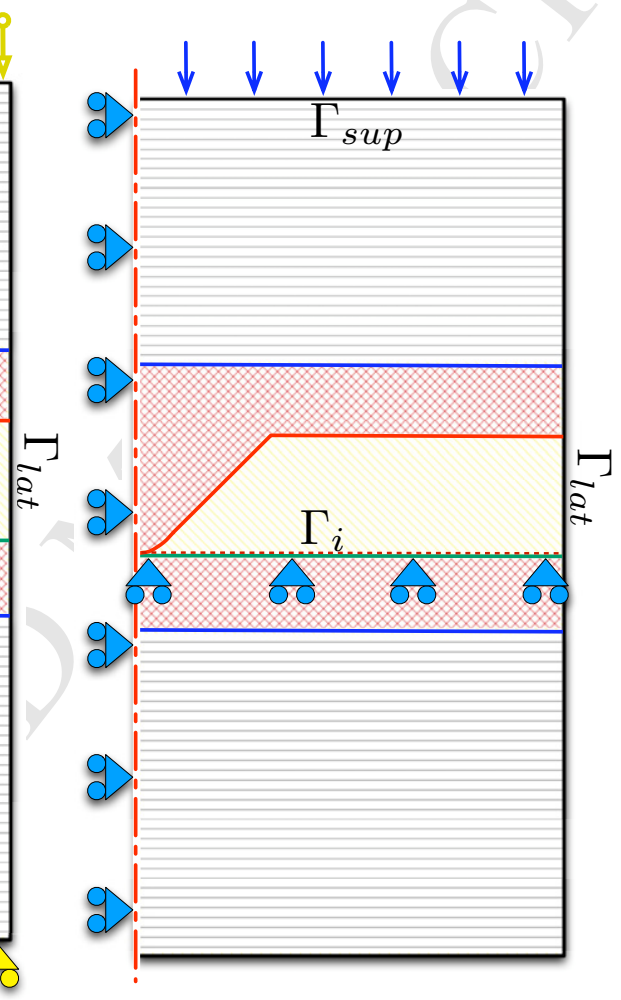

(b) Fluid mechanics.

Figure 4: Boundary conditions. 
condition. This problem describes the incompressible flow of polymer induced by the squeezing of the energy director, at the long observation time scale.

A homogeneous vertical stress distribution $\|s\|=6 \times 10^{6} \mathrm{~Pa}$ is imposed on the upper boundary and represents the typical holding force of the tool (Fig. 4b). The vertical velocity is imposed to zero on the internal boundary representing the upper surface of the lower plate in order to overcome the contact problems in the numerical simulation. It comes down to considering the lower plate as a rigid body.

\subsubsection{Thermal problem}

The thermal problem (T) given by

$$
\left\{\begin{array}{l}
\rho c\left(\frac{\partial T}{\partial t}+\nabla T \cdot v\right)=\nabla \cdot(k \nabla T)+Q \text { on }(\Omega) \\
Q=\frac{E^{\prime \prime} \omega}{2} \varepsilon: \varepsilon \text { on }(\Omega)
\end{array}\right.
$$

with the boundary conditions

$$
\left\{\begin{array}{l}
T(t=0)=T_{0} \text { on }(\Omega) \\
k . \nabla T \cdot \boldsymbol{n}=0 \text { on }\left(\Gamma_{\text {lat }} \cup \Gamma_{0} \cup \Gamma_{\text {sup }}\right)
\end{array},\right.
$$

governs the time-averaged temperature $T$ in the director. It is a classical convection conduction problem with an original thermal source $Q$. This power source results from the vibration effects. As shown in (Levy et al., 2010), the mechanical viscoelastic behaviour reduces to an elastic behaviour for quick loadings and can therefore be modelled with the elastic problem (1) and (2). Nevertheless the heating comes from the small viscous effect and is important because the pulsation $\omega$ of the loading is important. With a sinusoidal loading, the dissipated power averaged over an ultrasonic cycle is 
classically modeled, with the loss modulus $E^{\prime \prime}$, as above (Tolunay et al., 1983; Benatar and Gutowski, 1989; Wang et al., 2006; Suresh et al., 2007).

$T_{0}=23{ }^{\circ} \mathrm{C}$ is the initial ambient temperature. The boundary conditions consist of insulating the system $\Omega$. It ensures that the increase of temperature in the system is only due to mechanical dissipation and not to heat conduction through boundaries.

\subsection{Material data}

Material data are adapted from the literature (Cogswell, 1992; Nicodeau, 2005). A shear-thinning Carreau constitutive law is assumed for the polymer behaviour:

$$
\eta=\eta_{0}\left(1+\left(\lambda_{c} D_{e q}\right)^{2}\right)^{\frac{m-1}{2}}
$$

where $\eta_{0}$ is the consistency, $\lambda_{c}$ is the Carreau characteristic time, $m$ is the Carreau index and $D_{e q}=\sqrt{2 \boldsymbol{D}: \boldsymbol{D}}$ is the equivalent strain rate. This nonlinear law gives a better description of the finite deformation of the polymer for large strains. The dependency to temperature is assumed to follow an Arrhenius law:

$$
\eta_{0}(T)=A \cdot \exp \left(\frac{E_{a}}{R T}\right)
$$

where $A$ is a material parameter, $E_{a}$ is the activation energy of the material and $R=8.31 \mathrm{JK}^{-1} \mathrm{~mol}^{-1}$ is the gas constant. Even if this law is unrealistic for temperature below the glassy temperature $T_{g}$, it will ensure a high viscosity at low temperature. The polymer then behaves as a rigid body. This is likely not to influence the flow in the hot zones where the viscosity drops. 
Table 1: Material parameters.

\begin{tabular}{|l|ccc|}
\hline & Polymer & Air & Composite \\
\hline \hline Young's modulus $E(\mathrm{~Pa})$ & eq. $(9)$ & $5 \times 10^{3}$ & $9.3 \times 10^{9}$ \\
Poisson coefficient $\nu$ & 0.4 & 0 & 0.2 \\
\hline viscosity coefficient $A\left(\mathrm{~Pa} . \mathrm{s}^{\mathrm{m}}\right)$ & $5.6 \times 10^{-3}$ & $2 \times 10^{-5}$ & 10 \\
activation energy $E_{a}(\mathrm{~J})$ & $7.44 \times 10^{4}$ & 0 & $7.44 \times 10^{4}$ \\
Carreau time $\lambda(\mathrm{s})$ & 1 & 0 & 1 \\
Carreau power $m$ & 0.54 & 1 & 1 \\
\hline thermal conductivity $k\left(\mathrm{Wm}^{-1} \mathrm{~K}^{-1}\right)$ & 0.24 & $2.6 \times 10^{-2}$ & 0.72 \\
thermal capacity $\rho c\left(\mathrm{Jm}^{-3} \mathrm{~K}^{-1}\right)$ & $1.3 \times 10^{6}+4500 T\left[{ }^{\circ} \mathrm{C}\right]$ & $10^{3}$ & $2.22 \times 10^{6}$ \\
\hline loss modulus $E^{\prime \prime}(\mathrm{Pa})$ & $20 \times 10^{6}$ & 0 & 0 \\
sonotrode pulsation $\omega\left(\mathrm{rad} . \mathrm{s}^{-1}\right)$ & $1.25 \times 10^{5}$ & & \\
\hline
\end{tabular}

The polymer Young's modulus dependence to temperature is adapted from the literature (Benatar and Gutowski, 1989; Goyal et al., 2006; Li, 1999) and fitted with the law:

$$
\begin{aligned}
E(\theta)= & 2.8 \times 10^{9}\left[0.5-\arctan \left(\frac{\theta\left[{ }^{\circ} \mathrm{C}\right]-T_{g}}{20}\right) / \pi\right] \\
& +1.6 \times 10^{8} \mathrm{~Pa}
\end{aligned}
$$

which ensures a high value for temperature below $T_{g}=143^{\circ} \mathrm{C}$ and low value above.

The mechanical parameters of the air are chosen such that it will neither influence the fluid nor the elastic problem. Its thermal parameters are chosen as the ones of static air. Its very low effusivity $\sqrt{k \rho c}$ compared to the polymer or the composite shows that it won't influence the thermal resolution in the vicinity of the air/matter interface. The composite is supposed to be isotropic with properties corresponding to transverse properties, since it will mainly be loaded in this direction.

All material parameters are given in table 1. 


\section{Numerical methods}

The difficulties of solving numerically such a problem are of two kinds. First it is a multiphysical formulation which implies two distinct mechanical problems and a thermal problem. Then a large geometry change occurs when the director is squeezed. In order to handle both features, original numerical methods are needed. The boundary value problems will be solved using the well known finite element method. In this idea, a new finite element code including original numerical methods is developed.

\subsection{Eulerian framework and level set method}

Because of the large geometry change, an Eulerian framework was retained. It consists in having a fixed mesh wherein the matter moves. On the one hand it avoids the mesh distortion difficulties linked to a Lagrangian approach, see for example the review by Dubois et al. (2009) for this issue. On the other hand, it is well adapted to the velocity formulation of fluid mechanics problem (3).

In this Eulerian framework, a specific method is needed in order to describe the geometry evolution. Many numerical tools exist. The Volume of Fluid method (Hirt and Nichols, 1981), for instance, consists in managing the matter fraction in each element. Despite its relative simplicity, this method allows to determine the position of the interface within one element only. We propose here to use the more precise level sets method. It consists in describing an interface position with a scalar field giving the algebraic distance to the interface (Sethian, 1999). Besides the precise description of the interface location, which is given by the 0 iso-value of the field, the data management is simplified because the level set field is stored as any physical field. One 


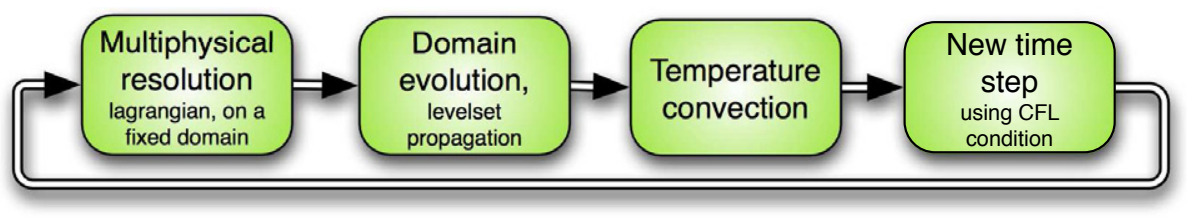

Figure 5: Flow chart of the proposed algorithm.

has to notice that the precision of the interface location, though more precise, remains limited to the size of the mesh at the vicinity of the interface. Therefore, the element size will define the precision of the numerical model.

Two level set fields allow to describe the polymer/air and polymer/composite interfaces. The material parameters used for integration only depend on the signs of the level sets. Elements crossed by the interfaces are cut for integration as in Moës et al. (1999).

The global resolution scheme is based on the operator splitting method (Smolianski, 2005). It consists in an explicit scheme as described in Fig. 5. At each time step four successive resolutions are performed:

1. The multiphysical problem is solved on a fixed geometry, using the iterative procedure discussed in the next section.

2. Using the solution of the flow problem, the geometry is updated. It is done by solving the convection equation for the level set fields $\phi$ over the whole domain containing composite, polymer and air:

$$
\frac{\partial \phi}{\partial t}+\nabla \phi \cdot \boldsymbol{v}=0
$$

This step is achieved using an Hamilton-Jacobi method (Sethian, 1999).

3. The temperature field, which is the only history variable in the problem, is convected in order to fit the geometry change:

$$
\frac{\partial T}{\partial t}+\nabla T \cdot \boldsymbol{v}=0
$$


This is achieved using an SUPG method (Brooks and Hughes, 1982).

4. The new time step $\delta t$ is determined such that the CFL condition

$$
\delta t<0.5 \frac{h_{e}}{\|v\|}
$$

is fulfilled in every element, $h_{e}$ being the element size and $\|v\|$ being a norm of the velocity field in the element. It prevents the interface from moving over more than one element in a time step and provides a stability condition for geometry changes (Courant et al., 1967).

\subsection{Multiphysical handling}

The physical fields are described in a finite element discretization framework as:

$$
\begin{aligned}
\boldsymbol{u} & =\left\{\boldsymbol{N}_{e}\right\}^{T}\left\{X_{e}\right\} \\
\boldsymbol{v} & =\left\{\boldsymbol{N}_{v}\right\}^{T}\left\{X_{v}\right\} \\
p & =\left\{N_{p}\right\}^{T}\left\{X_{p}\right\} \\
T & =\left\{\boldsymbol{N}_{T}\right\}^{T}\left\{X_{T}\right\}
\end{aligned}
$$

where $\left\{N_{i}\right\}^{T}$ are the interpolation functions and $\left\{X_{i}\right\}$ the unknown nodal values. For the sake of clarity, we define the concatenated vector $\left\{X_{f}\right\}^{T}=$ $\left\{\left\{X_{v}\right\}^{T}\left\{X_{p}\right\}^{T}\right\}$. The incompressibility condition in the flow problem (3) is ensured with a mixed velocity/pressure formulation. The associated LBB condition is fulfilled with a P1+/P1 MINI interpolation (Arnold et al., 1984). The interpolations used for the displacement and the temperature fields are linear per element (P1). It simplifies the data transfer between physics and minimizes the number of values stored at integration points.

In order to solve the three coupled problems simultaneously, we propose an algorithm based on the Gauss-Seidel method. If our physical problems 
were linear the global multiphysical problem could read

$$
[J]\{X\}=\{b\}
$$

where $\{X\}^{T}=\left\{\left\{X_{T}\right\}^{T}\left\{X_{e}\right\}^{T}\left\{X_{f}\right\}^{T}\right\}$ is the global concatenated unknown column vector, $\{b\}^{T}=\left\{\left\{b_{T}\right\}^{T}\left\{b_{e}\right\}^{T}\left\{b_{f}\right\}^{T}\right\}$ is the concatenation of the right hand side vectors representing the total residual to minimize for the coupled problem and

$$
[J]=\left[\begin{array}{ccc}
\frac{\partial b_{T}}{\partial X_{T}} & \frac{\partial b_{T}}{\partial X_{e}} & \frac{\partial b_{T}}{\partial X_{f}} \\
\frac{\partial b_{e}}{\partial X_{T}} & \frac{\partial b_{e}}{\partial X_{e}} & \frac{\partial b_{e}}{\partial X_{v}} \\
\frac{\partial b_{f}}{\partial X_{T}} & \frac{\partial b_{f}}{\partial X_{e}} & \frac{\partial b_{f}}{\partial X_{v}}
\end{array}\right]
$$

is the stiffness of the multiphysical problem. In our case where the multiphysical coupling are generally rather low, $J$ is diagonally dominant per block. Therefore, the Gauss-Seidel algorithm can be used per block to solve equation (14). It is a fixed point iterative method that consist in computing the series $\left\{X_{n}\right\}$ defined as

$$
(D-L)\left\{X_{n+1}\right\}=U\left\{X_{n}\right\}+\{b\}
$$

where $U$ is strictly upper triangular , $L$ strictly lower triangular $D$ diagonal and

$$
J=D-L-U
$$

The forward substitution of this algorithm consists in solving successively each physics using the already computed solutions. Our algorithm, derived from this method is applied to our non-linear problem. It consists in solving successively each non-linear problem until the three residuals converge (see 


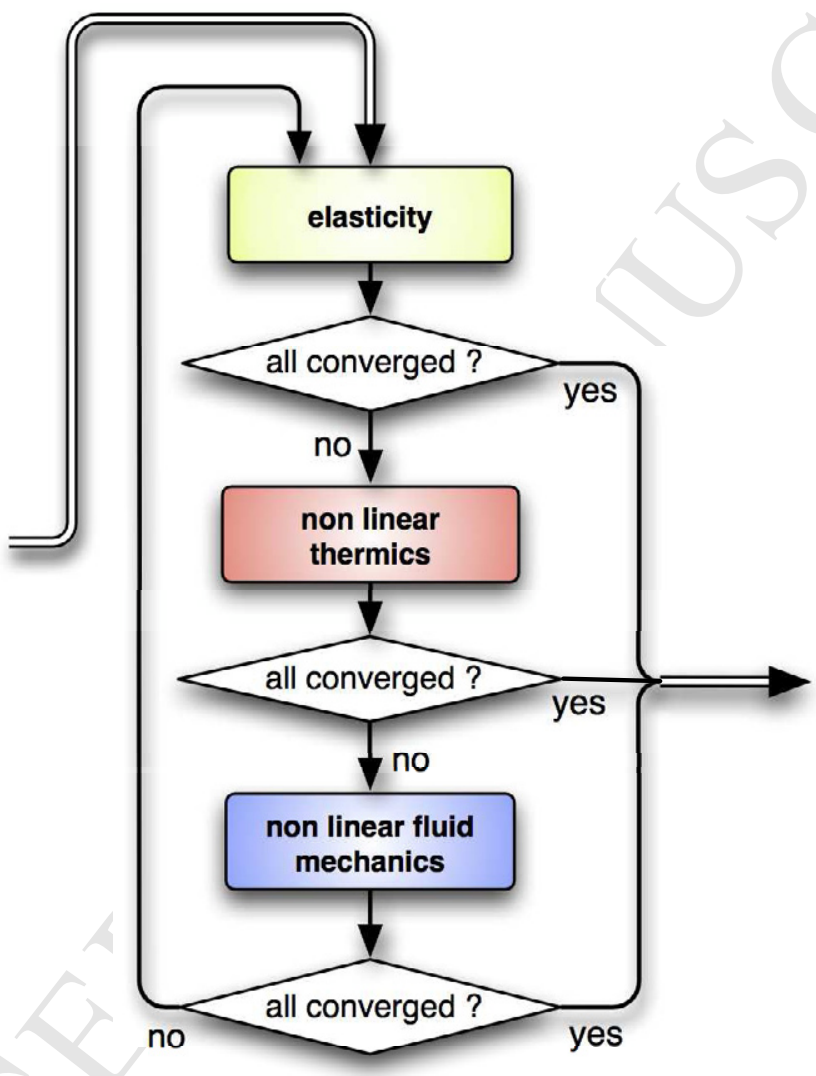

Figure 6: Iterative resolution for the multiphysical problem 
Fig. 6). The method is robust as soon as the multiphysical couplings are not too important. In practice two to three loops were needed. One should emphasize that even if the resolution is not done globally, if the convergence is reached, the rigorous global solution is obtained.

A Newton-Raphson algorithm is used for solving non-linear elementary physics. A line-search improvement (Zienkiewicz and Taylor, 1988) allows to accelerate the convergence, especially with fluid mechanics involving shearthinning non linear law (7).

The transient thermal conduction problem is solved using an implicit backward Euler scheme.

\subsection{Implementation}

The code is written in $\mathrm{C}++$ using the X-FEM library (Moës et al., 1999). Besides the interpolations and assembling tools, it contains classes for managing level sets and multi-material domains. Object oriented structure allows to handle multiphysical aspects quite easily: each physical formulation is derived from a generic class that sets a framework for a non-linear boundary value problem. Pre and post-processing are done using GMSH and solving of the linear system are done with SuperLU library.

\subsection{Mesh}

The basic retained mesh is made of 5244 unstructured triangles with a refinement at the initial position of the tip of the director, as shown on Fig. 7. A fillet is applied at the tip of the director, according to the real geometry, to a tenth of the director's height. The results shown thereafter focus on the welding zone. 


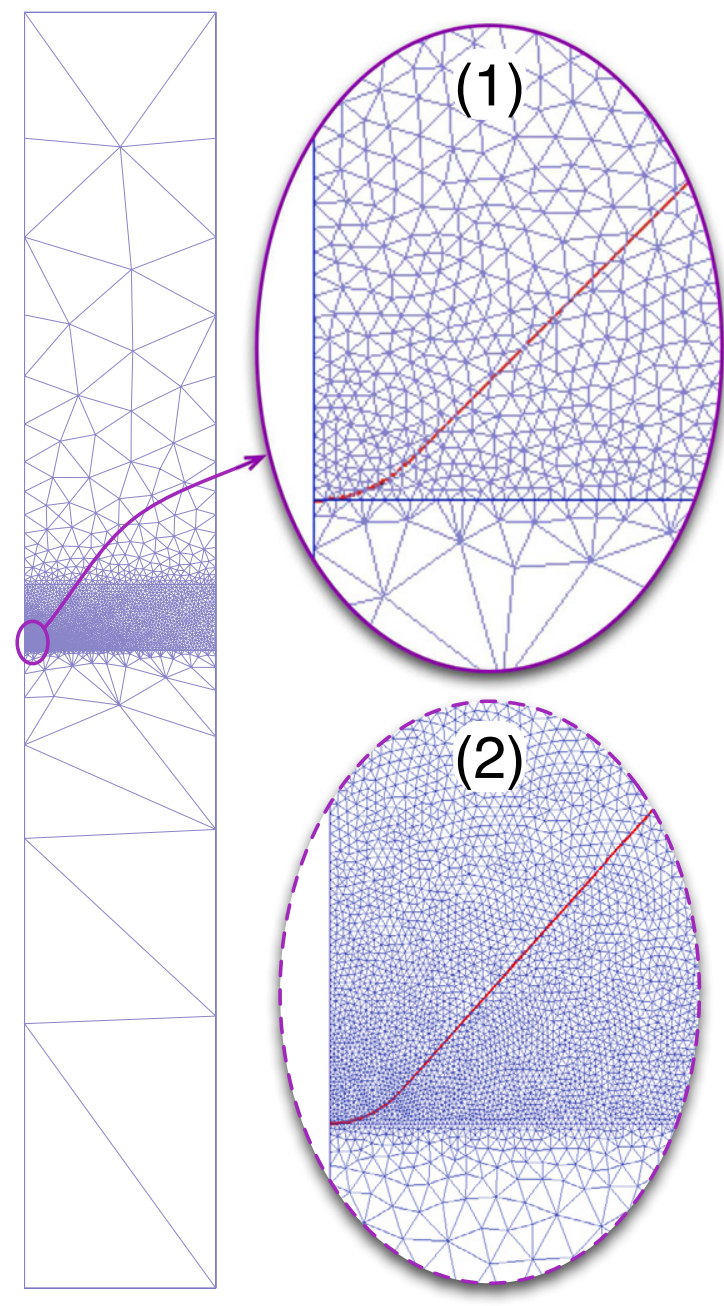

Figure 7: Meshes. (1) Reference mesh. (2) Finer mesh. 


\section{Qualitative validation}

Being the first simulation of simultaneous vibration and flow, it is still qualitative. In order to validate the orders of magnitude of the obtained results, we first propose a few comparisons to experiments.

\subsection{Heat flux}

Temperature measurements were done by incorporating thermocouples inside the plates. Considering a uni-dimensional model across the height of the plates, an inverse method allowed to determine a maximum macroscopic heat flux at the interface

$$
\phi_{\max }=155 \mathrm{~kW} \cdot \mathrm{m}^{-2} .
$$

To compare this value with the simulation, the heat flux at the mesoscopic scale of the energy director was averaged on the surface $y=0$ (see Fig. 2) of the lower plate. The evolution of this mean flux, given on Fig. 8, is in agreement with the measurement, even if the physical heating time evolution was not accessible from experiments.

\subsection{Thermal insulation}

Figure 10 shows that for time lower than $0.18 \mathrm{~s}$, the temperature gradient at positions $3 \times 10^{-3} \mathrm{~m}$ and $-3 \times 10^{-3} \mathrm{~m}$ are zero. The thermal boundary conditions of insulation, imposed on the thermal problem (5), are therefore validated, at least during this initial phase, when the director begins to flow.

\subsection{Energy}

The dissipated energy $E_{d}$ in the domain at time $t_{f}$ is equal to:

$$
E_{d}=\int_{0}^{t_{f}}\left(\int_{\Omega} \rho c \frac{\partial T}{\partial t} d v\right) d t .
$$




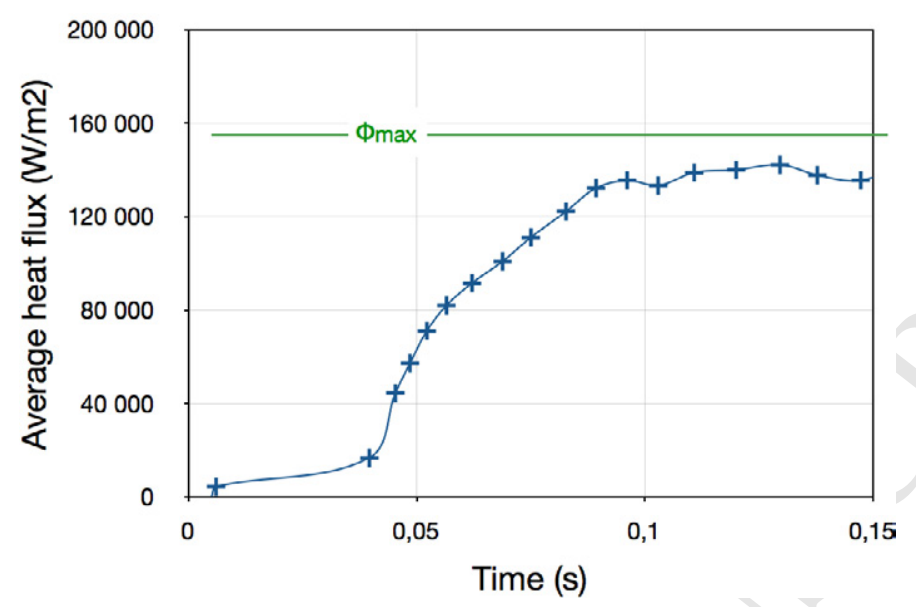

Figure 8: Averaged heat flux on the interface.

Approximating $\rho c$ with a constant $(\rho c)_{0}$ independent of temperature, the energy can be estimated as:

$$
E_{d} \sim(\rho c)_{0} \int_{\Omega}\left(T\left(t_{f}\right)-T_{0}\right)
$$

At an advanced stage, for time $t=0.14 \mathrm{~s}$, with $(\rho c)_{0}=1.3 \times 10^{6} \mathrm{JK}^{-1} \mathrm{~m}^{-3}$ for the polymer, we obtain

$$
E_{d} \sim 95 \mathrm{Jm}^{-1}
$$

The power is therefore around $650 \mathrm{~W}$ per linear meter of director, which is in agreement with the experimental test bed, delivering few hundreds of Watts.

\section{Discussion}

\subsection{Analysis of physical phenomena}

In this section we show that the proposed simulation allows to better understand the physical phenomena occurring during welding. 

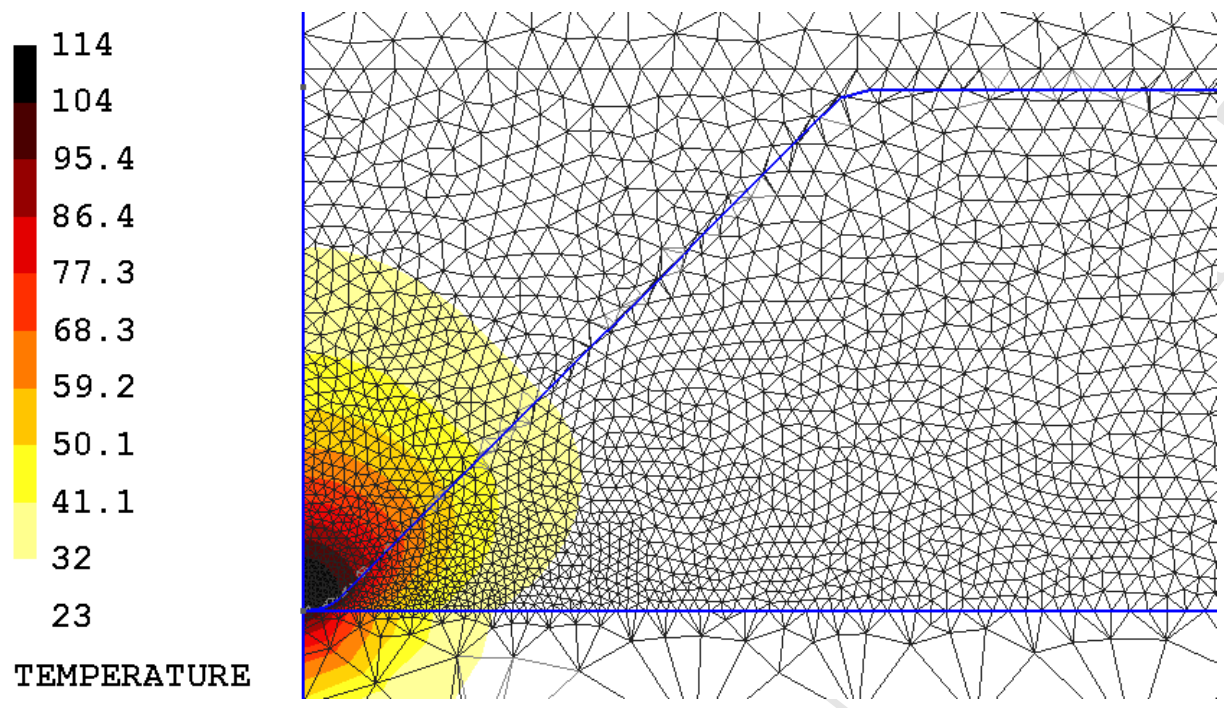

Figure 9: Temperature field simulated at time $t=0.03 \mathrm{~s}$.

\subsubsection{Localization}

During the initial phase of the simulation, as expected the triangular geometry of the director concentrates the strain. The source term in the thermal problem (5) being proportional to the square of the strain, the temperature raises quickly at the tip of the director, as shown on Fig. 9. This is the tip effect that confirms the ability of the director to concentrate energy. The goal of local heating at the interface is ensured as shown on Fig. 10 where the glass transition of PEEK (about $150^{\circ} \mathrm{C}$ ) is passed after a very short time $(\sim 0.05 \mathrm{~s})$.

\subsubsection{Fold}

Once the temperature is high enough, the viscosity drops at the tip of the director and leads to the formation of a fold. This fold flows as the upper plate is squeezed. It progressively fills the whole gap between the two plates, as shown on Fig. 11, where the surface is represented on the right hand side 


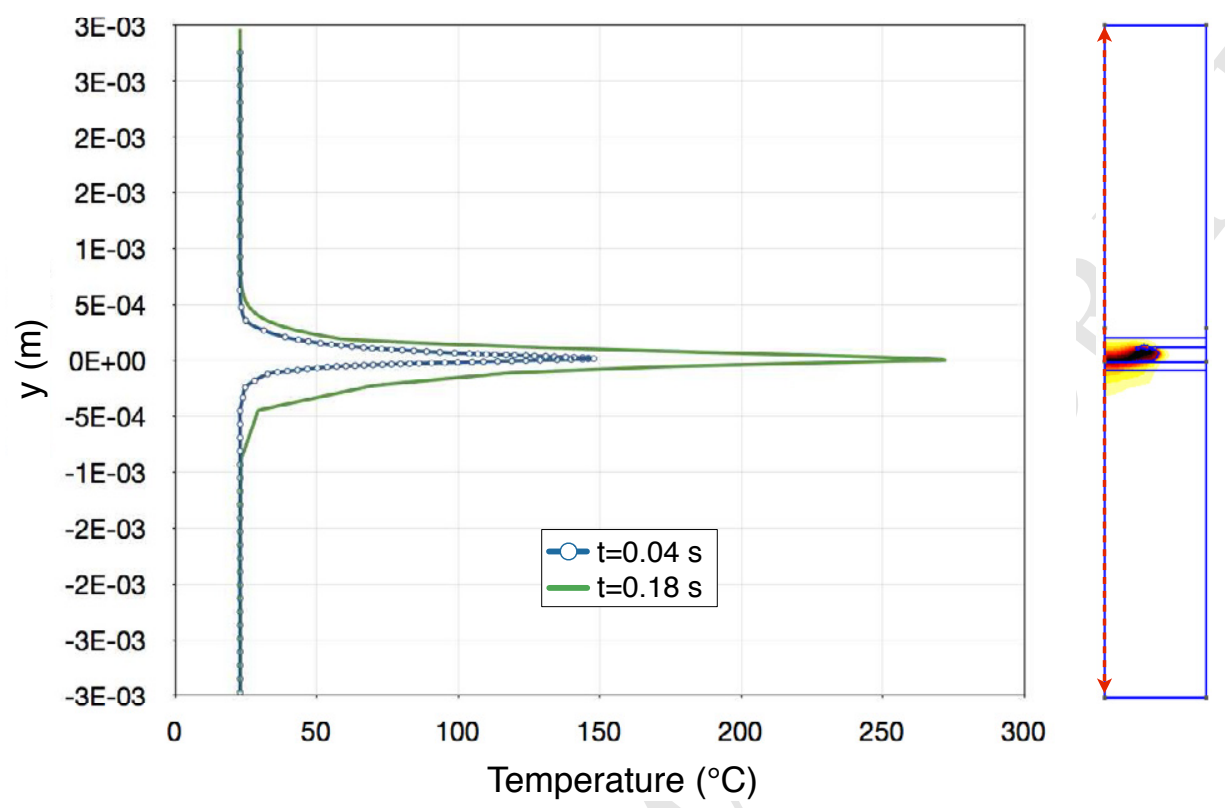

Figure 10: Temperature along the vertical axis of symmetry.

for different simulation times.

Figure 12 shows the comparison between the simulation and a photograph, made with an optical microscope, of a sample obtained on a stopped welding. The predicted morphology of the fold, is in agreement with the experiment. It therefore qualitatively validates the adopted description.

\subsubsection{Intimate contact and healing}

The above described fold morphology enables welding conditions. On the one hand, the flow along the lower plate ensures intimate contact. On the other hand, it provides a high temperature at the level of the interface which is an essential condition for healing. Qualifying adhesion is a complex problem involving several factors, such as temperature, time, morphology or pressure (Yang and Pitchumani, 2002). The adoption of a criterion might 


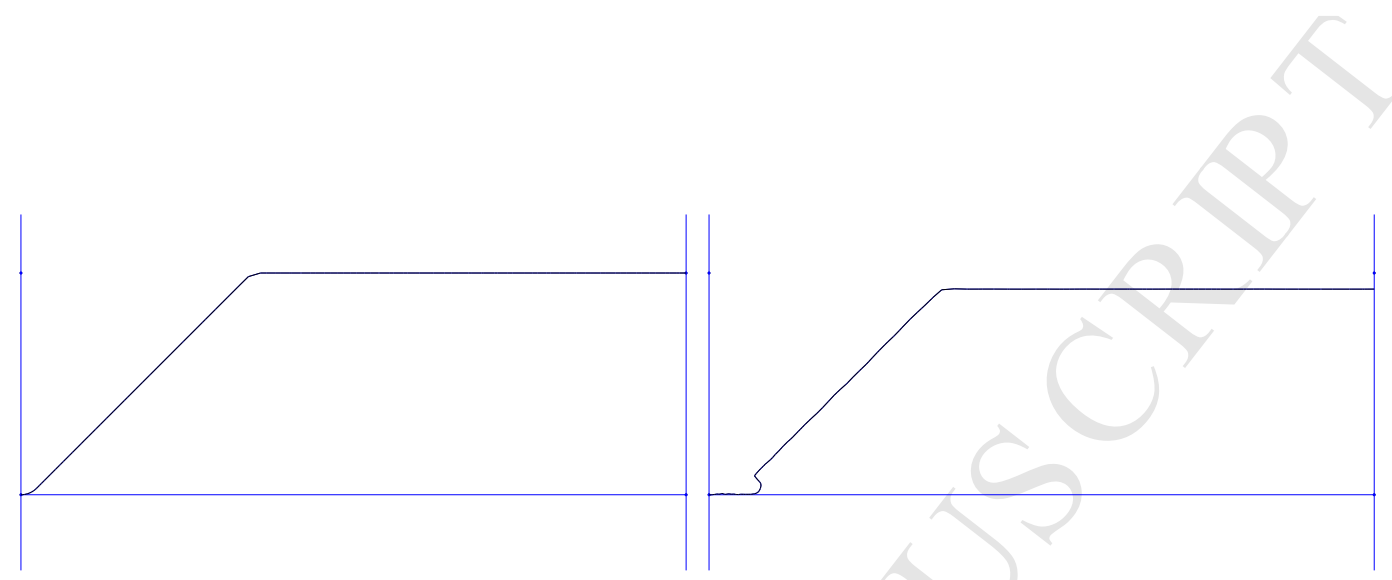

(a) initial time

(b) $t=0.05 \mathrm{~s}$

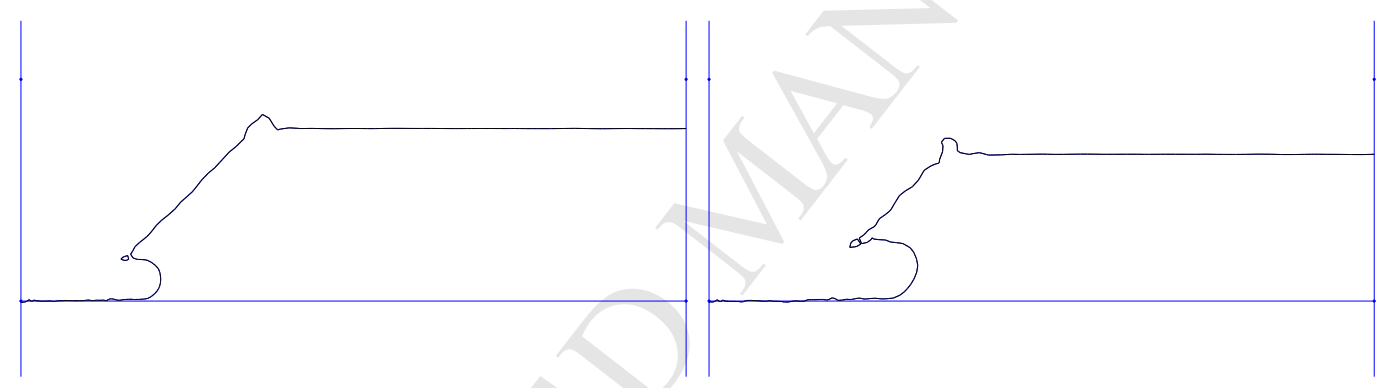

(c) $t=0.08 \mathrm{~s}$

(d) $t=0.10 \mathrm{~s}$

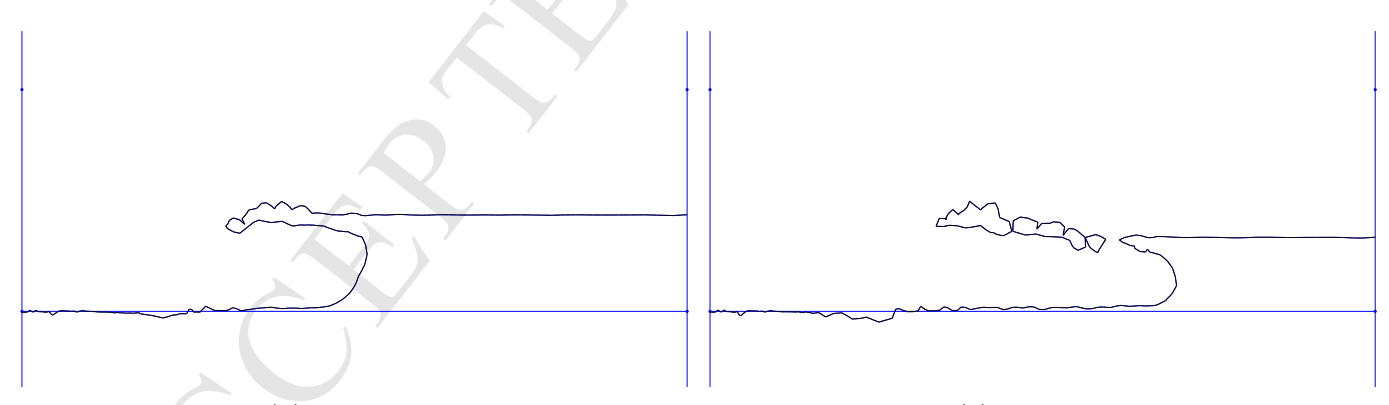
(e) $t=0.15 \mathrm{~s}$
(f) $t=0.20 \mathrm{~s}$

Figure 11: Free surface evolution, initiation and expansion of the fold. 


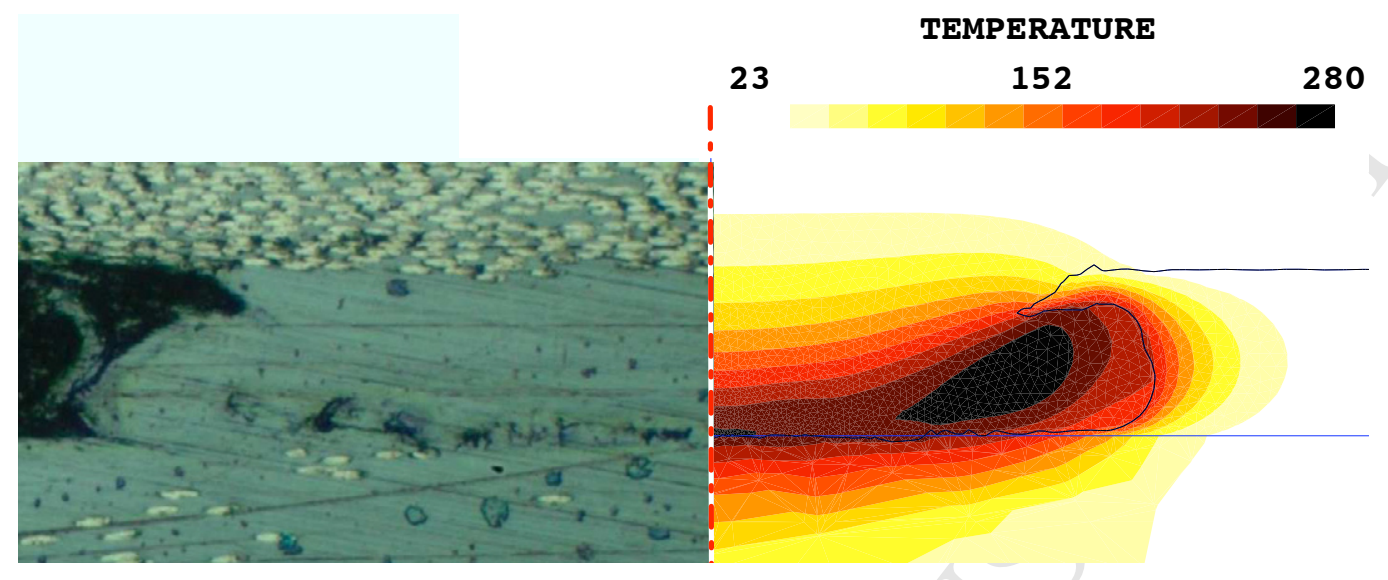

Figure 12: Comparison between a picture on a stopped experiment and the reference simulation (temperature field at time $t=0.12 \mathrm{~s}$ ).

help evaluating the quality of the welding. This criterion may be determined by post-processing simulation such as the one presented.

\subsection{Level-set and Eulerian framework}

The Eulerian approach with tracking of the free surface is quite original for a solid structure simulation. In the present process, it is justified by the important deformation occurring in the fold. Indeed a Lagrangian approach would have required special care because of elements that would got highly distorted. The present simulations show that the proposed strategy enables to cope with strains as high as about 100\%. Nevertheless it presents some limitations discussed hereunder.

First the Eulerian framework imposes a fluid approach for the modeling. Therefore, the traction condition on the upper boundary is not imposed on a physical surface because of the inward velocity, but on a spatial fixed surface. It results in adding some matter to the system $\Omega$. In our particular case, the geometry is such that the boundary are far enough from the processing zone 
(cf Fig. 2). It ensures that:

1. In the flow problem (3), the added matter is close to room temperature and behaves as a rigid body. Therefore, applying the boundary condition on this geometrical boundary $\Gamma_{\text {sup }}$ will not affect the solution about the energy director.

2. The matter displacement during the whole process will not exceed the initial height of the gap between the plate $h=0.3 \mathrm{~mm}$. The thicknesses of the plates $(2 \times 3 \mathrm{~mm} \sim 20 \times h)$ is important enough to ensure that the added matter creates only a negligible change of height. Moreover, as for the flow problem, the added matter on the far boundary $\Gamma_{\text {sup }}$ is close to room temperature, and much more rigid than the material in the processing zone. In conclusion, the added matter does not affect the elastic problem (1) significantly.

3. The thermal insulation conditions in the thermal problem (5) ensures that no energy is added to the system with the added matter. Furthermore, as clear from Fig.10, the duration of the process is small enough so that the heat does not reach the upper nor the lower boundaries. Actually, in such a case, the thermal boundary condition has almost no effect on the results around the energy director.

In Fig. 11, one may notice that the polymer/interface is penetrating in the lower plate especially at an advanced stage. There are two explanations for this penetration, which nevertheless is never bigger than one element. First, the explicit procedure illustrated in Fig. 5 may induce penetration, only limited with the CFL condition (12). Then the Hamilton Jacobi propagation algorithm needs a normal speed at each node. The normal to the level set 


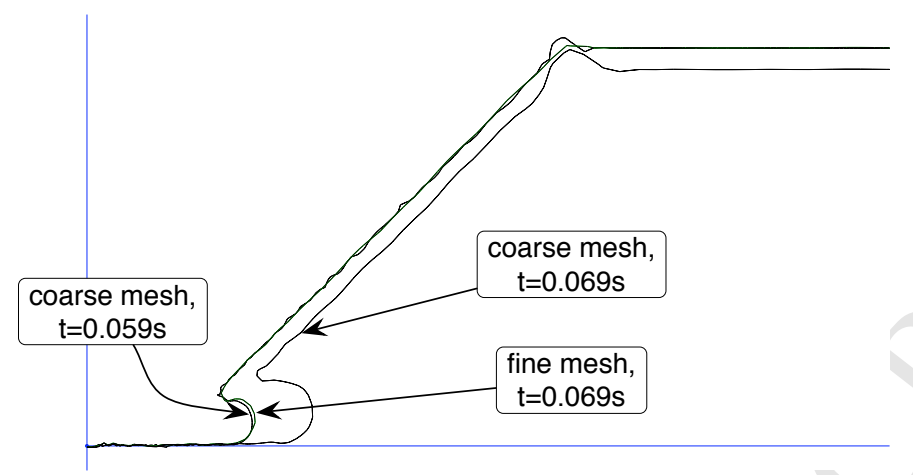

Figure 13: Comparison of the free fold morphology simulated with the fine mesh and the coarse mesh.

is its gradient, but the level set field interpolation being linear, the normal is constant per element. Its value at a node is considered to be the average of each neighbouring element. This induces an artifact in the normal velocity computation. This penetration may be limited by penalizing it (Levy et al., 2009) or simply by refining the mesh since, as already mentioned, the penetration never exceeds one element.

\subsection{Mesh dependency}

A simulation was done using a finer mesh made of 32030 triangles as shown on Fig. 7. Figure 13 shows that the free surface simulated using the fine mesh at time $t=0.069 \mathrm{~s}$ is similar to the one obtained with a coarse mesh at time $t=0.059 \mathrm{~s}$. The simulated flow with a coarse mesh is therefore in advance compared to the finer mesh.

In order to confirm this delay between the two simulations, the relative temperature difference $\left(T_{f}-T_{c}\right) / T_{f}$ is represented on Fig. 14b. $T_{f}$ is the temperature simulated with the fine mesh at time $t=0.069 \mathrm{~s}$ and $T_{c}$ with the reference coarse mesh at time $t=0.059 \mathrm{~s}$ for which the deformation 


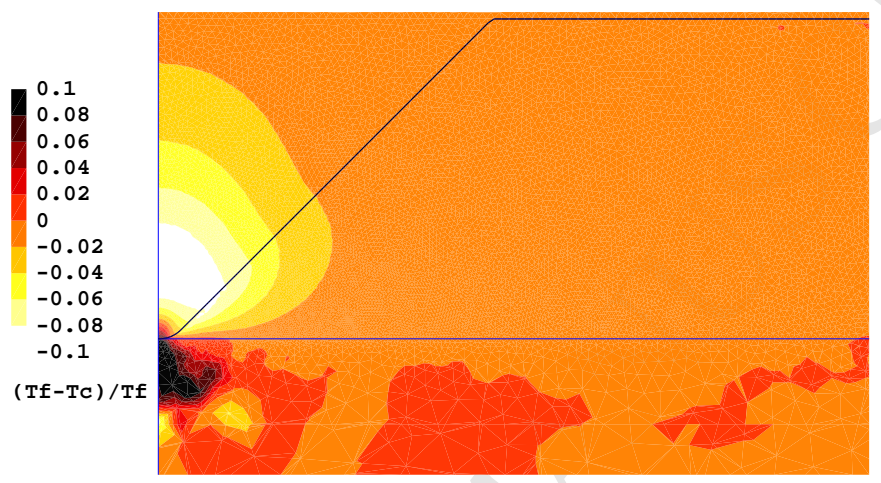

(a) near initial time, at $t=0.01 \mathrm{~s}$.

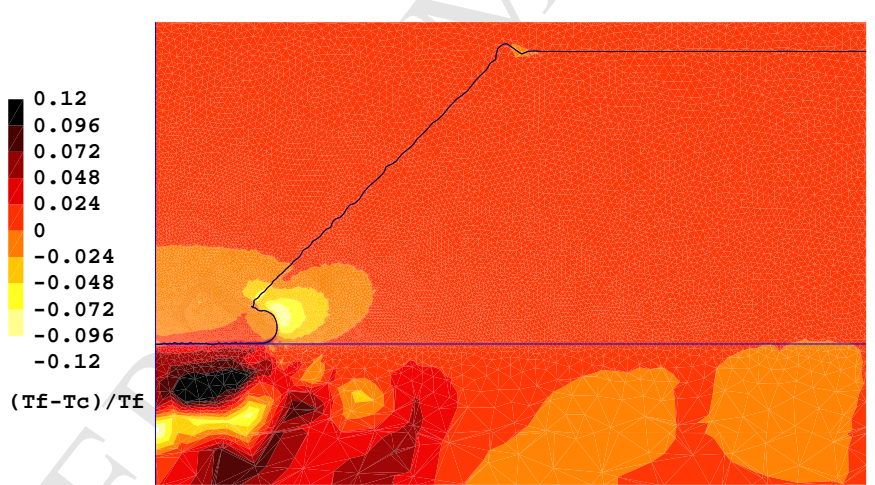

(b) at $t=0.069 \mathrm{~s}$ for the $T_{f}$ and $t=0.059 \mathrm{~s}$ for $T_{c}$.

Figure 14: Relative temperature difference between fine and coarse mesh. 
is similar. The figure shows a maximum difference of about $10 \%$, mainly located at the tip of the director. In the coarse mesh simulation, the flow is in advance because the initial heating at the tip of the director is higher. This can be partly explained by the fact that the coarse mesh induces a poorer contact between the director and the lower plate. The director is therefore more thermally insulated and heats faster, as shown on Fig. 14a.

This difference confirms that the description of a complex geometry, such as the fillet at the tip of the director, using level set would be improved with a finer mesh. With this aim, an automatic algorithm may be used to refine mesh at the level of the free surface. Such an algorithm is currently under development. Additionally, it may allow to better track the evolution of a porosity during the flow of the polymer.

\subsection{Limitations for high coupling case}

The proposed numerical tool allows to solve the multiphysical problem rigorously since when the iterative solver converged, every residual is converged. Nevertheless in some cases, the iterative algorithm may not converge. If the physics are highly coupled the matrix $[J]$ in equation $(15)$ is not diagonally dominant anymore. An illustration of this limit is obtained when the holding force of the sonotrode is lowered. When the temperature raises at the tip of the director the flow and the formation of the fold described above is not that fast and the localisation becomes more important. Multiphysical coupling are not weak anymore and the iterative solver does not converge. Two solutions would allow to get round this limit. One may solve the full multiphysical problem using the fully assembled stiffness matrix $[J]$ or modify the iterative solver, taking into account the cross sensitivities 
$\partial b_{i} / \partial X_{j}, i \neq j$ of eq. (15) in the Gauss Seidel procedure.

\section{Conclusion}

This work focuses on the process of ultrasonic welding of thermoplastic composites. It proposes a modeling and a simulation strategy for the flow of polymer at the interface. The developed simulation tool fully handles the multiphysical aspects of the process that is to say heat transfer, vibration and flow. The original choice of using the level set method in an Eulerian framework was shown to be relevant for this problem; it allows to treat the large deformation of the energy director. Predictions of the code were qualitatively validated by fitting experimental order of magnitude. The novel simulation results allow to understand the physical phenomena that ensure welding: the tip effect (thermomechanical localization) and the creation of a fold of polymer that fills the gap between the two plates.

Nevertheless, even if the obtained results are already satisfactory, the carried mesh analyses showed that improvements would be obtained by an appropriate mesh adaptation technique. An automatic mesh refinement tool will be added to our code. It should also enable to investigate the $3 \mathrm{D}$ effect.

Indeed, it is now important to understand the "continuous welding" and the tridimensional effect induced by the advance of the sonotrode along the welding zone.

\section{Acknowledgements}

We address our thanks to Eric Soccard from EADS IW who supported this work and provided his experimental and industrial knowledge of the process. 


\section{References}

Arnold, D., Brezzi, F., Fortin, M., 1984. A stable finite element for the stokes equations. Calcolo 21, 337-344.

Benatar, A., Eswaran, R., Nayar, S., 1989. Ultrasonic welding of thermoplastics in the near-field. Polymer Engineering and Science 29, 1689-1698.

Benatar, A., Gutowski, T.G., 1989. Ultrasonic welding of peek graphite apc-2 composites. Polymer Engineering and Science 29, 1705.

Brooks, A.N., Hughes, T., 1982. Streamline upwind/petrov-galerkin formulations for convection dominated flows with particular emphasis on the incompressible navier-stokes equations. Computer Methods in Applied Mechanics and Engineering 32, 199-259.

Cogswell, F., 1992. Thermoplastic aromatic polymer composites. Woodhead.

Courant, R., Friedrichs, K., Lewy, H., 1967. On the partial difference equations of mathematical physics. IBM Journal of Research and Development $11,215$.

Dubois, C., Le Corre, S., Zarroug, M., Rozicki, P., Moës, N., 2009. Impact on highly compressible media in explicit dynamics using the $\mathrm{x}$-fem. Computational Mechanics 46, 329-348.

Goyal, R., Tiwari, A.N., Mulik, U.P., Negi, Y.S., 2006. Dynamic mechanical properties of $\mathrm{Al}_{2} \mathrm{O}_{3} /$ poly(ether ether ketone) composites. Journal of Applied Polymer Science 104, 568-575. 
Grewell, D.A., Benatar, A., Park, J.B., 2003. Plastics and Composites Welding Handbook. Hanser.

Hirt, C., Nichols, B., 1981. Volume of fluid/vof/ method for the dynamics of free boundaries. Journal of Computational Physics 39, 201-225.

Levy, A., Le Corre, S., Poitou, A., Chevaugeon, N., 2009. Développement d'un code éléments finis pour simuler le soudage par ultrasons de matériaux composites., in: 16èmes Journées Nationales des Composites (JNC 16), AMAC, Toulouse, France. http://hal.archives-ouvertes.fr/hal-00387409/.

Levy, A., Le Corre, S., Poitou, A., Soccard, E., 2010. Ultrasonic welding of thermoplastic composites, modeling of the process using time homogenization. International Journal for Multiscale Computational Engineering In press.

Li, R., 1999. Time-temperature superposition method for glass transition temperature of plastic materials. Materials Science and Engineering A $278,36-45$.

Moës, N., Dolbow, J., Belytschko, T., 1999. A finite element method for crack growth without remeshing. International Journal for Numerical Methods in Engineering 46, 131-150.

Nicodeau, C., 2005. Modélisation du Soudage en Continu des composites a Matrice Thermoplastique. Ph.D. Thesis. Ecole Nationale Superieure d'Arts et Métiers de Paris. http://pastel.paristech.org/1506/.

Nonhof, C., Luiten, G., 1996. Estimates for process conditions during the 
ultrasonic welding of thermoplastics. Polymer Engineering and Science 36, 1177.

Sethian, J., 1999. Level Set Methods and Fast Marching Methods: Evolving Interfaces in Computational Geometry, Fluid Mechanics, Computer Vision, and Materials Science. Cambridge University Press.

Smolianski, A., 2005. Finite-element / level-set / operator-splitting (FELSOS) approach for computing two-fluid unsteady flows with free moving interfaces. International Journal for Numerical Methods in Fluids 48, 231269.

Suresh, K., Roopa Rani, M., Prakasan, K., Rudramoorthy, R., 2007. Modeling of temperature distribution in ultrasonic welding of thermoplastics for various joint designs. Journal of Materials Processing Technology 186, $138-146$.

Tolunay, M.N., Dawson, P.R., Wang, K.K., 1983. Heating and bonding mechanisms in ultrasonic welding of thermoplastics. Polymer Engineering and Science 23, 726-733.

Wang, X., Yan, J., Li, R., Yang, S., 2006. FEM investigation of the temperature field of energy director during ultrasonic welding of peek composites. Journal of Thermoplastic Composite Materials 19, 593.

Yang, F., Pitchumani, R., 2002. Healing of thermoplastic polymers at an interface under nonisothermal conditions. Macromolecules 35, 3213-3224.

Zienkiewicz, O., Taylor, R., 1988. The finite element method: Solid and 
fluid mechanics dynamics and non-linearity. volume 2. McGraw-Hill Book Company Europe. 